\title{
Teens, vaccines and the age of consent
}

\author{
Cite as: CMAJ 2019 March 25;191:E348-9. doi: 10.1503/cmaj.109-5730
}

Posted on cmajnews.com on Mar. 7, 2019.

W hen should doctors start talking to teenage patients about the risks and benefits of vaccines? As unvaccinated children become adults, teens requesting vaccination against their parents' wishes, perhaps influenced by events like the recent measles outbreak in British Columbia, have made headlines in Canada and the United States.

Some provinces have an "age of medical consent" when children can make their own medical decisions. It ranges from 14 in Quebec to 16 in Saskatchewan or New Brunswick. Other provinces, including Ontario and Quebec, have no set age.

Regardless of whether a province has a suggested age of medical consent, however, the "mature minor rule" applies, explains Dr. Mike Dickinson, past president of the Canadian Paediatric Society. That means a teen can consent to a vaccine if they're mature enough to understand its risks and benefits. "More important than the age number is the developmental level of the child and their decision-making capacity," says Dickinson. "One clue might be other reasonable decisions the young adult has made in their life; another might be how they're functioning at school."

In the case of vaccines, teenagers can consent at an earlier age, arguably, than for complex medical interventions such as a surgery, says Timothy Caulfield, a Canada Research Chair in Health Law and Policy at the University of Alberta. "The risks are relatively low, and the benefits are easy to understand, so I think a teenager can be quite young and still completely competent to consent on their own to vaccines."

Caulfield suggests that doctors ask unvaccinated teenagers about their views on vaccination and provide information rather than wait for them to bring it up.

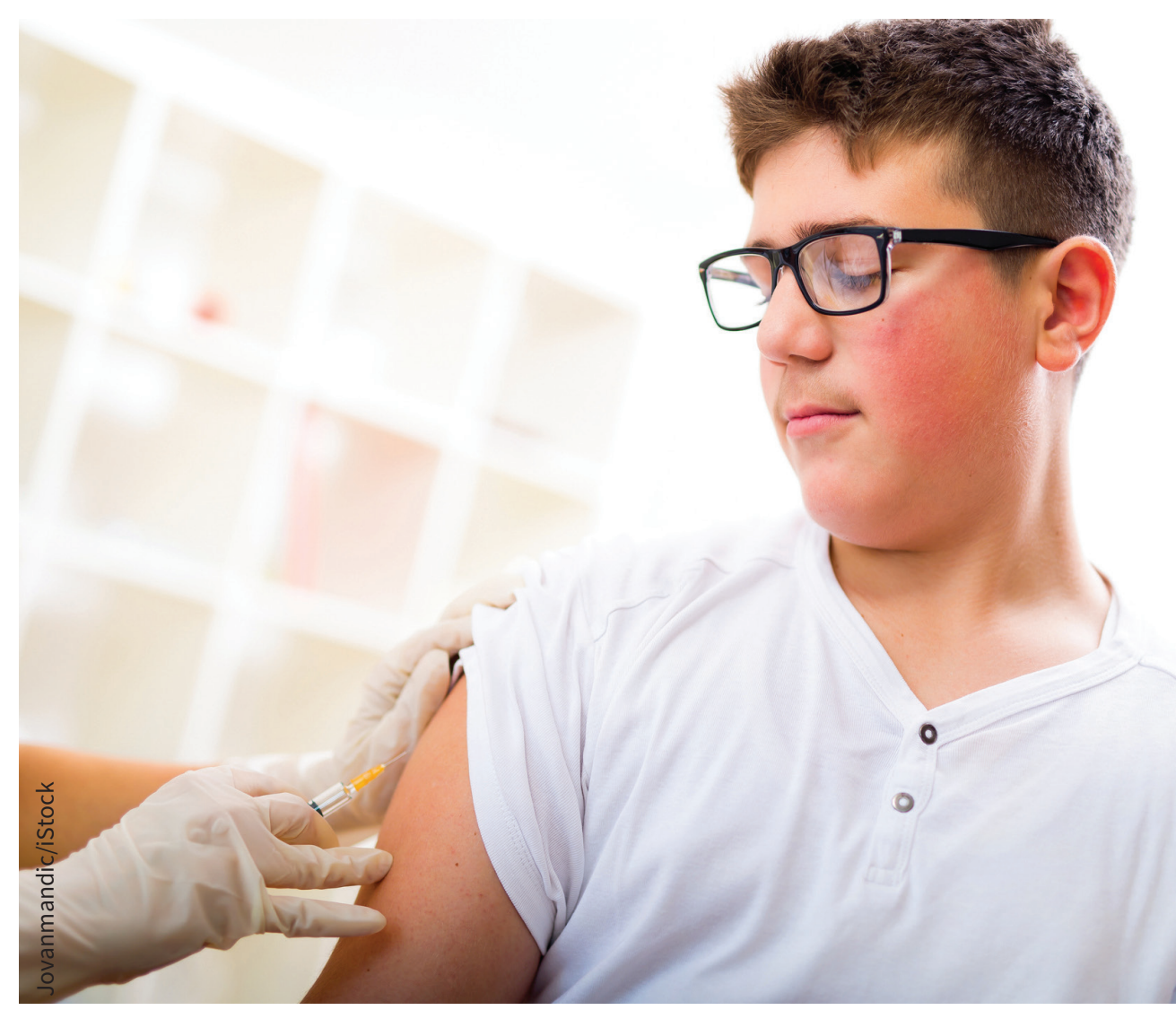

Teenagers can consent to vaccination if they are mature enough to understand the risk and benefits.

"As a thought experiment, you could imagine a teen saying, 'You knew I wasn't vaccinated. You knew I was competent to make that decision. How come you didn't tell me about it?"'

Generally speaking, doctors involve teenagers in conversations about vaccination starting in Grade 9, when they are 13 or 14. For example, Vancouver Coastal Health still sends consent forms to parents for Grade 9 vaccinations, but a nurse will vaccinate a teenager deemed to understand the risks and benefits and who wishes to be vaccinated, even if the form was misplaced or indicates parents don't consent.
The nurse will, however, give the student an opportunity to call a parent to let them know, because "we don't want to create a rift between the child and parent," explains Dr. Meena Dawar, a medical health officer for Vancouver Coastal Health. But it's rare that a child wants to receive a vaccine against their parents' wishes. This situation occurs fewer than five times a year in the Vancouver Coast Health region, says Dawar.

Dickinson agrees with this approach. Pediatricians like there to be an "open line of communication" between teenagers and their parents, but "every family situation is unique, and we would tend to follow the teen's lead if for whatever rea- 
son they didn't feel safe or they didn't want to disclose it to the parent," he says.

Dawar doesn't think more teenagers are vaccinating themselves against their parents' wishes because of BC's measles outbreak, although it may have led some parents to reconsider their stance. "We have had a few families who have been refusing vaccines in the past come forward to have their children immunized in the current outbreak," she says.
Given that teens can consent to vaccinations, there should be awareness campaigns aimed at preteens, similar to antismoking campaigns that focused on this age group in the past, says Dr. Kumanan Wilson, a senior scientist in the clinical epidemiology program at the Ottawa Hospital Research Institute.

"This is a fundamental issue to their health, and in a few years, they'll have that ability to make that decision for themselves," he says. "There are an increasing number of adolescent vaccinations occurring as well."

By their teenage years, youth may not be as interested in information about vaccination. "The optimal age to sensitize them is the preteen period," says Wilson. "Once they become teenagers, they want to have their own opinions on things."

Wendy Glauser, Toronto, Ont. 